Article

\title{
Performance Analysis of the Shore-to-Reef Atmospheric Continuous-Variable Quantum Key Distribution
}

\author{
Shujing Zhang ${ }^{1}$, Chen Xiao ${ }^{2}$, Chun Zhou ${ }^{1}$, Xiang Wang ${ }^{1}$, Jianshu Yao ${ }^{3}$ and Hailong Zhang ${ }^{1, *}$ \\ and Wansu Bao ${ }^{1}$ \\ 1 Henan Key Laboratory of Quantum Information and Cryptography, SSF IEU, Zhengzhou 450001, China; \\ zhangsjsjzhang@163.com (S.Z.); zc@qiclab.cn (C.Z.); dixonwx@163.com (X.W.); bws@qiclab.cn (W.B.) \\ 2 SSF IEU, Zhengzhou 450001, China; xc1090@sohu.com \\ 3 Fujian Chengyi College, Jimei University, Xiamen 361021, China; yaojianshu0351@163.com \\ * Correspondence: zhhl049@126.com; Tel.: +86-15699320860
}

Received: 30 October 2019; Accepted: 3 December 2019; Published: 4 December 2019

check for updates

\begin{abstract}
The effects of sea salt concluded in oceanic atmosphere are ubiquitous in practical wireless optical links. Here a shore-to-reef atmospheric continuous-variable quantum key distribution (CVQKD) model is established on the basis of Mie scattering theory, with the aim to characterize the complex case of beam propagation in the atmosphere caused by sea salt particles. The effects on performance of shore-to-reef atmospheric CVQKD under the sea salt particles and relative humidity are also studied. Simulation results show that the increase of particle radius and relative humidity will lead to the degeneration of secret key rate. Extending the channel distance also reduces the secret key rate. This paper provides a basis for the establishment of practical shore-to-reef atmospheric CVQKD model. The research of this paper also gives momentous reference for the study of optical communication channel models with other suspended particles over the ocean.
\end{abstract}

Keywords: sea salt particles; relative humidity; atmospheric CVQKD; secret key rate

\section{Introduction}

Quantum communication has witnessed rapid development. Quantum key distribution (QKD) [1,2] is no longer applicable to minor scales and short distances with favorable ambient conditions. Traditional optical fiber channel QKD [3,4] has not yet met the growing demand, hence atmospheric channel QKD [5-7] has attracted increasing attention. Compared to optical fiber channel QKD with fixed-attenuation, atmospheric channel QKD could cover a wider range of transmission with more flexible transmission [8,9]. For example, it is difficult to apply optical fiber channels for quantum communication on the sea, so atmospheric channels have come into play. However, the environments of atmosphere are relatively complicated, and beam propagation in the atmosphere is inevitably affected by the atmosphere [10-12]. The influence of complex atmosphere on beam propagation mainly involves absorption and scattering of beam by atmospheric molecules and aerosols and disturbance of beam by atmospheric turbulent motion. The former mainly leads to the loss of beam energy, with the name of atmospheric attenuation. The latter causes beam wandering deformation and scintillation $[13,14]$, commonly referred to as atmospheric turbulence effect.

The influence of complex atmosphere on QKD protocol performance cannot be elided. There is some research on it. For example, satellite-sea surface-receiver plane model, underwater QKD model, and air-to-water channel model [15-18] were proposed for QKD protocol in the sea area. However, there is no complete study on the effect of sea salt on atmospheric continuous-variable quantum key distribution (CVQKD). In fact, compared with metropolitan atmospheric channel, the sea salt content 
of the ocean atmospheric channel increases, which has a remarkable effect on the beam transmission and further affects the performance of the protocol. Therefore, for future experiments and applications, it is of great necessity to analyze the effects of sea salt particles on atmospheric CVQKD.

The purpose of this paper is to obtain a model of shore-to-reef atmospheric channel CVQKD via exploring the atmospheric attenuation caused by suspended sea salt particles. It is momentous to study the effect of sea salt on the performance of atmospheric channel CVQKD, which ensures the normal implementation of the protocol in high concentration of sea salt atmospheric environment. The extinction effect caused by absorption and scattering of beam by sea salt particles is the main factor affecting the quantum transmission with the large concentration of sea salt. Therefore, based on the characteristics of sea salt particles and Mie scattering theory $[19,20]$, this paper establishes a sea salt aerosol model and explores the relationship between the extinction factor and the radius of sea salt particles. The results reveal that the extinction factor increases with the growth of the radius of sea salt particles. Then, the study applies the model to the atmospheric channel CVQKD model. Two cases of fixed shore-based distance or sea surface distance is simulated and analyzed, respectively. We find that the secret key rate lessens with the increase of the particle radius, regardless of fixed shore-based distance or fixed sea surface distance. The increase of relative humidity will lead to the decline of secret key rate but the decrease is less than that of particle radius. The research hopes to provide theoretical basis and evaluation support for the implementation of atmospheric channel CVQKD protocol in high concentration of sea salt environment.

This paper is organized as follows. In Section 2, ocean atmospheric CVQKD model is established. In Section 3, we describe the atmospheric turbulence effect. In Section 4, a sea salt aerosol model is established to illustrate the extinction effect of sea salt particles on the grounds of characteristics of sea salt particles and Mie scattering theory. In Section 5, the performance of the CVQKD protocol is simulated and analyzed. Finally, conclusions are given in Section 6.

\section{Shore-to-Reef Atmospheric CVQKD}

In this section, we analyze the secret key rate of atmospheric CVQKD. A shore-to-reef atmospheric CVQKD protocol is shown in Figure 1. This model is based on the model in ref [8]. In this paper, we use a different channel. It is composed of shore-based atmospheric channel and oceanic atmospheric channel. Einstein-Podolsky-Rosen (EPR) source generates an entangled state with variance V. The $B_{0}$ is one mode of the entangled state that is transmitted to Bob through a fading channel, which is depicted by a distribution of transmittance $T$, and Bob performs the heterodyne detection to measure the quadrature. The detection efficiency $\eta$ is used to depict the imperfection of the detector and the electronic noise $v_{e l}$ contained in variance $v$ [8].

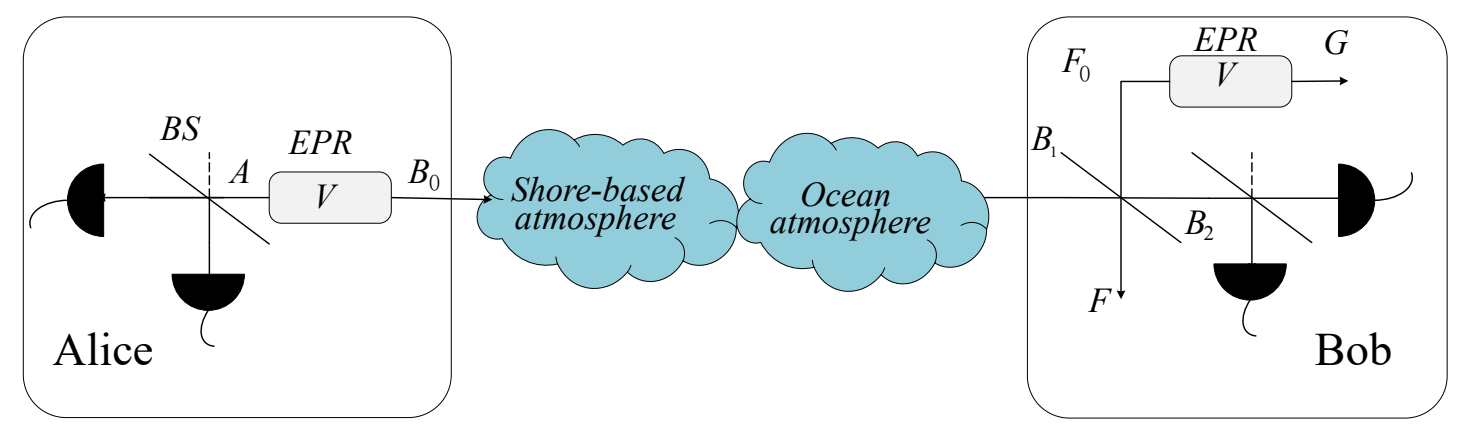

Figure 1. Continuous-variable quantum key distribution (CVQKD) through shore-to-reef atmospheric channel.

The secret key rate $K$ is given as [21]

$$
K=\beta_{1} I_{A B}-\chi_{B E},
$$


where $\beta_{1}$ is the reconciliation efficiency, $I_{A B}$ is the Shannon mutual information of Alice and Bob, and $\chi_{B E}$ is the Holevo quantity, which can be expressed as [22]

$$
\chi_{B E}=S\left(\rho_{E}\right)-\int d m_{B} p\left(m_{B}\right) S\left(\rho_{E}^{m_{B}}\right),
$$

where $m_{B}$ represents measurement of Bob, $p\left(m_{B}\right)$ represents the probability density of the measurement, $\rho_{E}^{m_{B}}$ represents the state of eavesdropper conditional on the measurement result of Bob, and $S(\cdot)$ represents the Von Neumann entropy.

Then, we should calculate $I_{A B}$ and $\chi_{B E}$ in order to obtain the secret key rate. The covariance matrix of a two-mode squeezed vacuum state with variance [22]:

$$
\gamma_{A B}=\left(\begin{array}{cc}
V I & \sqrt{V^{2}-1} \sigma_{z} \\
\sqrt{V^{2}-1} \sigma_{z} & V I
\end{array}\right),
$$

where $V$ represents Alice's modulation variance, $\sigma_{z}=\operatorname{diag}(1,-1)$ and $I=\operatorname{diag}(1,1)$.

On the assumption that the state is described by probability distribution $\left\{p_{i}\right\}$ of transmittance $\left\{T_{i}\right\}$ through a fluctuating channel, and we can use the mean value of transmittance $<T>$ and the mean of the square root of transmittance $<\sqrt{T}>$ to replace the fluctuating transmittance [7], the covariance matrix can be expressed as:

$$
\gamma_{A B}^{\prime}=\left(\begin{array}{cc}
V I & <\sqrt{T}>\sqrt{V^{2}-1} \sigma_{z} \\
<\sqrt{T}>\sqrt{V^{2}-1} \sigma_{z} & <T>(V+1 /<T>-1+\varepsilon) I
\end{array}\right) .
$$

Now, we can read the mutual information as [8]:

$$
I_{A B}=\log _{2} \frac{1}{1-\frac{<\sqrt{T}>^{2}(V-1)}{<T>(V+\chi)}},
$$

where $\chi=2\left(1+v_{e l}\right) / \eta<T>-1+\varepsilon$. Furthermore, $\chi_{B E}$ can also be obtained [21]:

$$
\chi_{B E}=\sum_{i=1}^{2} G\left(\frac{\lambda_{i}-1}{2}\right)-\sum_{i=3}^{5} G\left(\frac{\lambda_{i}-1}{2}\right),
$$

where $G(x)=(x+1) \log _{2}(x+1)-x \log _{2}(x)$.

$\lambda_{1,2}$ could be calculated as

$$
\lambda_{1,2}^{2}=\frac{1}{2}\left[A \pm \sqrt{A^{2}-4 B}\right],
$$

where [8]

$$
\begin{aligned}
& A=V^{2}\left(1-2<\sqrt{T}>^{2}\right)+2<\sqrt{T}>^{2}+<T>^{2}(V+1 /<T>-1+\varepsilon)^{2} \\
& B=\left[V^{2} \operatorname{var}(\sqrt{T})+<\sqrt{T}>^{2}+<T>V(1 /<T>-1+\varepsilon)\right]^{2}
\end{aligned}
$$

$\operatorname{var}(\sqrt{T})=\langle T\rangle-\langle\sqrt{T}\rangle^{2}$ is the variance of $\sqrt{T}, \lambda_{3,4,5}$ are the symplectic eigenvalues of covariance matrix $\gamma_{A F G^{\prime}}^{m^{B}} \gamma_{A F G}^{m^{B}}=\gamma_{A F G}-\gamma_{h}$. Furthermore, $\gamma_{h}=\sigma_{A F G B_{2}}^{T} H \sigma_{A F G B_{2}}$. For heterodyne $H^{\text {het }}=\left(\gamma_{B_{3}}+\right.$ $I)^{-1}[8]$.

$$
\gamma_{A F G B_{3}}=\left(\begin{array}{cc}
\gamma_{A F G} & \sigma_{A F B_{2}}^{T} \\
\sigma_{A F G B_{2}} & \gamma_{B_{3}}
\end{array}\right) \text {. }
$$


When we simplify the results of equation $\gamma_{A F G}^{m^{B}}=\gamma_{A F G}-\gamma_{h}$, the elements of Equation (4) can be defined as [8]

$$
\begin{aligned}
& a=V \\
& b=<\sqrt{T}>\sqrt{V^{2}-1} \\
& c=<T>(V+1 /<T>-1+\varepsilon)
\end{aligned}
$$

then we can obtain [8]

$$
\gamma_{A F G}=\left(\begin{array}{ccc}
a I & b \sqrt{1-\eta} \sigma_{z} & \mathbf{0} \\
b \sqrt{1-\eta} \sigma_{z} & {[(v-c) \eta+c] I} & \sqrt{\eta\left(v^{2}-1\right)} \sigma_{z} \\
\mathbf{0} & \sqrt{\eta\left(v^{2}-1\right)} \sigma_{z} & v I
\end{array}\right) .
$$

For heterodyne case [8]

$$
\gamma^{\text {het }}=\frac{1}{(c-v) \eta+v+1}\left(\begin{array}{ccc}
\gamma_{1-1}^{\text {het }} & \gamma_{2-1}^{\text {het }} & \gamma_{3-1}^{\text {het }} \\
\gamma_{2-1}^{\text {het }} & \gamma_{2-2}^{\text {het }} & \gamma_{3-2}^{\text {het }} \\
\gamma_{3-1}^{\text {het }} & \gamma_{3-2}^{\text {het }} & \gamma_{3-3}^{\text {het }}
\end{array}\right)
$$

where

$$
\begin{aligned}
& \gamma_{1-1}^{\text {het }}=b^{2} \eta I \\
& \gamma_{2-2}^{\text {het }}=(c-v)^{2} \eta(1-\eta) I \\
& \gamma_{3-3}^{\text {het }}=(1-\eta)\left(v^{2}-1\right) I \\
& \gamma_{2-1}^{\text {het }}=b \eta(c-v) \sqrt{1-\eta} \sigma_{z} \\
& \gamma_{3-1}^{\text {het }}=-b \sqrt{\eta(1-\eta)\left(v^{2}-1\right)} I \\
& \gamma_{3-2}^{\text {het }}=-(c-v)(1-\eta) \sqrt{\eta\left(v^{2}-1\right)} \sigma_{z}
\end{aligned}
$$

where $v=1+2 v_{e l} /(1-\eta)$. Combining Equations (11), (12), and $\gamma_{A F G}^{m^{B}}=\gamma_{A F G}-\gamma_{h}$, we can get $\lambda_{3,4,5}[8]$ :

$$
\lambda_{3,4}^{2}=\frac{1}{2}\left[C \pm \sqrt{C^{2}-4 D}\right]
$$

$\lambda_{5}=1$. Specifically, $C$ and $D$ can be shown as [8]

$$
\begin{aligned}
& C=\frac{A \chi^{2}+2 \chi(a \sqrt{B}+c)+B+2 b^{2}+1}{(c+\chi)^{2}} . \\
& D=\left(\frac{a+\sqrt{B} \chi}{c+\chi}\right)^{2}
\end{aligned} .
$$

It is notable that the transmittance consists of transmittance of share-based atmosphere and ocean atmosphere; therefore, we can show the transmittance as $T=T_{s a} T_{o a}$.

\section{Atmospheric Turbulence Effect}

Atmospheric turbulence has the characteristics with irregularity and randomness. In the turbulence, the temperature, velocity, pressure, refractive index, and other physical properties fluctuate randomly along with time. Atmospheric turbulence is the main factor that restricts the performance of free-space optical communication. It will lead to beam wandering and beam broadening. Ultimately, it will result in the reduction of the reliability and stability for the whole communication system.

When the beam propagates in turbulent atmosphere, the diameter or area of the spot received by the aperture will increase and the center of the spot will deflect at the receiving plane, which is the phenomenon of beam wandering and broadening. The geometry for this model is shown in Figure 2. Where, $a$ is the radius of receiver, $W$ is the radius of beam, and $r_{w}$ is the distance of wandering [9]. 


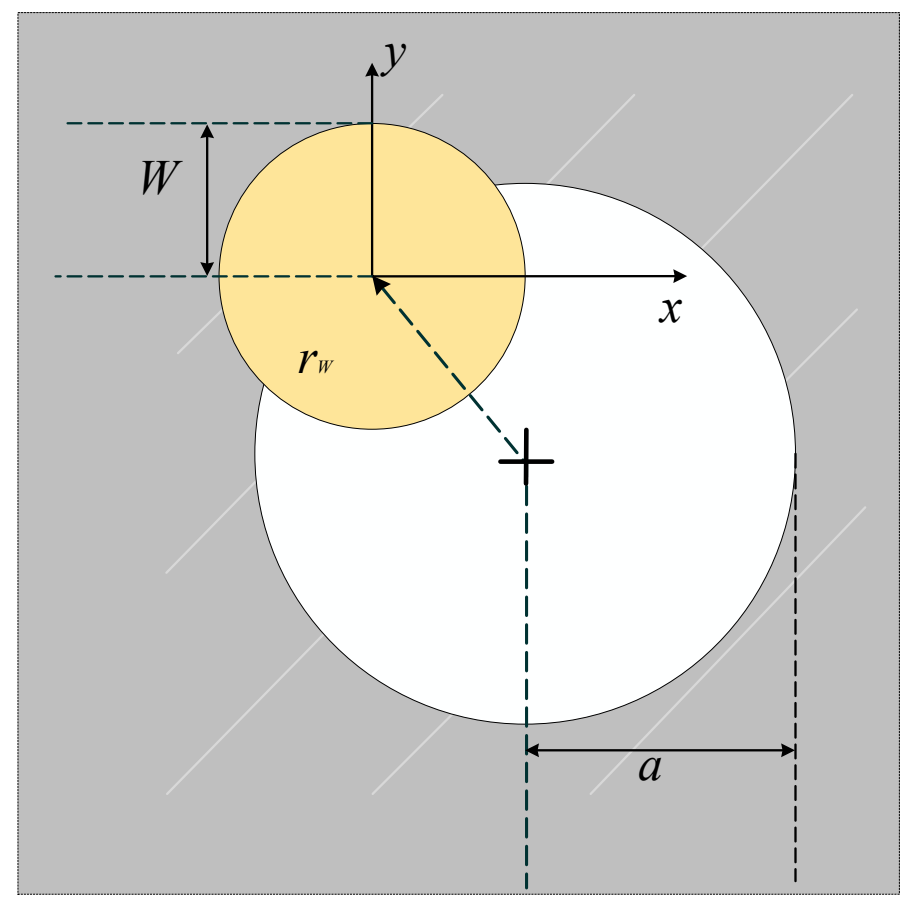

Figure 2. Beam wandering and broadening.

Assuming that the beam fluctuates in the central plane of the receiver aperture, the probability density distribution can be expressed by Weibull distribution [9]:

$$
P\left(T_{1}\right)=\frac{2 R^{2}}{\sigma^{2} Q T_{1}}\left(2 \ln \frac{T_{0}}{T_{1}}\right)^{\frac{2}{Q}-1} \exp \left(-\frac{1}{2 \sigma^{2}} R^{2}\left(2 \ln \frac{T_{0}}{T_{1}}\right)^{\frac{2}{Q}}\right) .
$$

$\sigma^{2}$ is variance of wandering:

$$
\sigma^{2}=1.919 C_{n}^{2} L^{3}\left(2 W_{0}\right)^{-1 / 3},
$$

where $L$ is the distance of transmission and $W_{0}$ is the beam spot radius at the radiation source. $C_{n}^{2}$ is the index of refraction structure function. In this paper, we use the Hufnagel-Valley model for $C_{n}^{2}$. It is a height distribution model of $C_{n}^{2}$ proposed based on experimental measurement that is closely related to height. This model includes stratosphere and troposphere and is widely used [18],

$$
C_{n}^{2}=0.00594\left(V_{w} / 27\right)^{2}\left(h / 10^{-15}\right)^{10} e^{-h / 1000}+2.7 \times 10^{-16} e^{-h / 1500}+A e^{-h / 100},
$$

where $V_{w}$ is the wind speeds, $h$ is the altitudes, and $A$ is constant. Usually, we set it as $1.7 \times 10^{-14}$.

$R$ is scale parameter, $Q$ is shape parameter [9],

$$
\begin{gathered}
R=a\left[\ln \left(\frac{2 T_{0}^{2}}{1-\exp \left(-4 \frac{a^{2}}{W^{2}}\right) I_{0}\left(4 \frac{a^{2}}{W^{2}}\right)}\right)\right]^{-\frac{1}{Q},} \\
Q=8 \frac{a^{2}}{W^{2}} \frac{\exp \left(-4 \frac{a^{2}}{W^{2}}\right) I_{1}\left(4 \frac{a^{2}}{W^{2}}\right)}{1-\exp \left(-4 \frac{a^{2}}{W^{2}}\right) I_{0}\left(4 \frac{a^{2}}{W^{2}}\right)} \times\left[\ln \left(\frac{2 T_{0}^{2}}{1-\exp \left(-4 \frac{a^{2}}{W^{2}}\right) I_{0}\left(4 \frac{a^{2}}{W^{2}}\right)}\right)\right]^{-1} .
\end{gathered}
$$

$I_{i}$ is modified Bessel function and $T_{0}$ is the maximum transmission efficiency for a given beam radius $W$, which can be written as [9] :

$$
T_{0}^{2}=1-\exp \left(-2 \frac{a^{2}}{W^{2}}\right)
$$


The transmission efficiency of the beam with a beam radius $W$ on the receiving plane can be expressed as follows [9]:

$$
T_{1}^{2}=T_{0}^{2} \exp \left(-\left(\frac{r}{R}\right)^{Q}\right) .
$$

Figure 3 shows the relationship between refractive index structure function and wind speeds. It can be seen clearly from the figure that the higher the altitude, the greater the influence of refractive index structure function on wind speed. At altitude of 0 and $1 \mathrm{~km}$, the refractive index structure function is almost unchanged with wind speed. In addition, the lower the altitude, the larger the refractive index structure function, which indicates that severe atmospheric fluctuations could cause drastic turbulence effects. When the altitude is above $10 \mathrm{~km}$, the refractive index structure function is very small, and the atmospheric turbulence effect can be negligible.

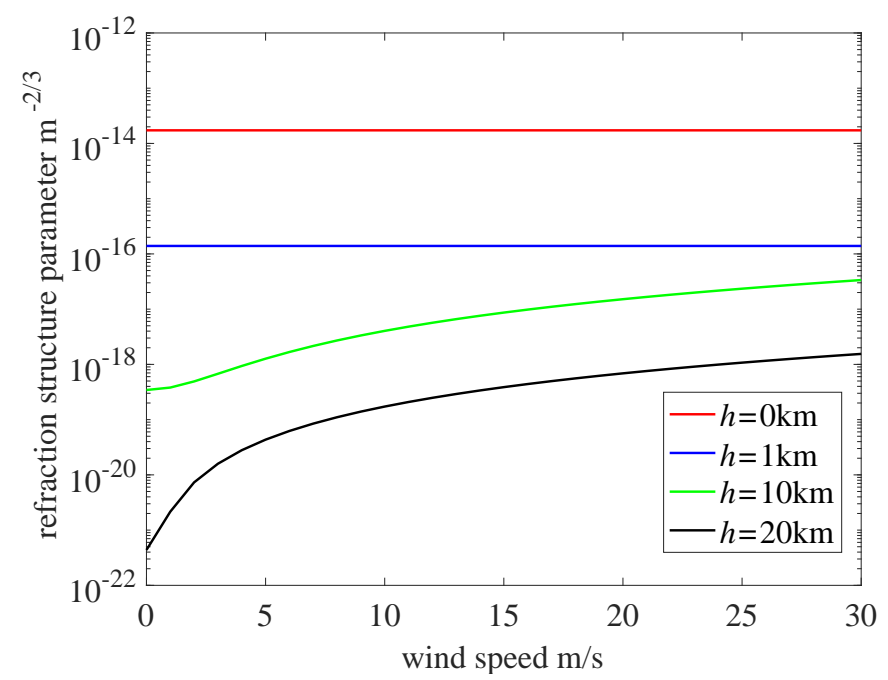

Figure 3. The refraction structure at altitudes of $h=0 \mathrm{~km}, h=1 \mathrm{~km}, h=10 \mathrm{~km}$, and $h=20 \mathrm{~km}$ with various wind speeds.

\section{Extinction Effect of Sea Salt Particles}

Sea salt particles are mainly produced by the tremendous transpiration of the ocean surface and the breakdown of the spoondrift [19]. The radius, relative humidity, and concentration of sea salt particles in ocean atmospheric channel cause absorption and scattering to beam and then affect the performance of CVQKD protocol. In this section, we establish a model of sea salt aerosol based on the characteristics of sea salt particles and Mie scattering theory.

We definite $x=\frac{2 \pi a}{\lambda}$, where $a$ is the radius of particle and $\lambda$ is the wave length. When $x \ll 1$, we can use Rayleigh scattering to describe the scattering. When $x \approx 1$, we can use Mie scattering theory to solve the problem of scattering. The scattering of light in the atmosphere is mainly Mie scattering. Some suspended particles in nature for atmosphere can be regarded as spherical particles and Mie scattering theory is used for research. Mie scattering considers that the size of particles and the refractive index of materials have a decisive influence on the scattering and absorption of light [23-25].

In the analysis, sea salt particles are supposed to be spherical particles. When a plane wave is incident on a single spherical particle, based on the single scattering theory, the case of an isotropic homogeneous spherical particle is considered. The electric field components of the incident wave in the vertical and parallel scattering planes are connected with the corresponding components of the incident beam via the scattering matrix [19]:

$$
\left(\begin{array}{c}
E_{V}^{S} \\
E_{H}^{S}
\end{array}\right)=\frac{\exp (i k r)}{-i k r}\left(\begin{array}{cc}
S_{1} & S_{3} \\
S_{4} & S_{4}
\end{array}\right)\left(\begin{array}{c}
E_{V}^{i} \\
E_{H}^{i}
\end{array}\right)
$$


where $k=2 \pi / \lambda, \lambda$ is incident wave length and $S_{i}$ is scattering matrix. The scattering matrix value is determined by the particle shape, scale, and refractive index. Due to the symmetry of spherical particles, $S_{3}=0, S_{4}=0$. After a complex solution $S_{1}, S_{2}$ are expressed as follows:

$$
\begin{aligned}
& S_{1}=\sum_{n=1}^{\infty} \frac{2 n+1}{n(n+1)}\left[a_{n} \pi_{n}+b_{n} \tau_{n}\right] \\
& S_{2}=\sum_{n=1}^{\infty} \frac{2 n+1}{n(n+1)}\left[b_{n} \pi_{n}+a_{n} \tau_{n}\right] .
\end{aligned}
$$

$\pi_{n}=\frac{P_{n}^{(1)}(\cos \theta)}{\sin \theta}=\frac{d P_{n}(\cos \theta)}{d(\cos \theta)}, \tau_{n}=\frac{d P_{n}^{(1)}(\cos \theta)}{d(\cos \theta)}$, is the coefficient of scattering angle, where $P_{n}^{(1)}(X)$ is the first kind of Legendre function. The coefficient of the Mie scattering $a_{n}, b_{n}$ can be expressed:

$$
\begin{aligned}
& a_{n}=\frac{\psi_{n}(x) \psi_{n}^{\prime}(y)-m \psi_{n}^{\prime}(x) \psi_{n}(y)}{\xi_{n}(x) \psi_{n}^{\prime}(y)-m \xi_{n}^{\prime}(x) \psi_{n}(y)} \\
& b_{n}=\frac{m \psi_{n}(x) \psi_{n}^{\prime}(y)-\psi_{n}^{\prime}(x) \psi_{n}(y)}{m \xi_{n}(x) \psi_{n}^{\prime}(y)-\xi_{n}^{\prime}(x) \psi_{n}(y)}
\end{aligned}
$$

where $\psi_{n}(X)=\sqrt{\frac{\pi X}{2}} J_{n+\frac{1}{2}}(X), \xi_{n}(X)=\sqrt{\frac{\pi X}{2}} H_{n+\frac{1}{2}}(X), J_{n+\frac{1}{2}}(X), H_{n+\frac{1}{2}}(X)$ is the first kind of semi-integral order Bessel function and Hankel functions of the second kind of semi-integral order, respectively. $\psi_{n}^{\prime}(X), \xi_{n}^{\prime}(X)$ is the derivative of $\psi_{n}(X), \xi_{n}(X)$, respectively. $m$ is the refractive index of sea salt, $m=m_{r e}+i m_{i e}$. The refractive index of wet sea salt particles is determined by both dry particles and condensed liquid water [19]:

$$
\begin{aligned}
& m_{r e}=m_{r w}+\left(m_{r r}-m_{r w}\right)\left[\frac{r(r h)}{r_{0}}\right]^{-3} \\
& m_{i e}=m_{i w}+\left(m_{i w}-m_{i w}\right)\left[\frac{r(r h)}{r_{0}}\right]^{-3},
\end{aligned}
$$

$m_{r w}, m_{i w}, m_{r r}, m_{i r}$ is the real and imaginary parts of refractive index of water and dry sea salt particles, respectively. The refractive index of seawater is usually taken as 1.33 . The refractive of dry sea salt particles is usually taken as $1.55-i 0.0035 . \frac{r(r h)}{r_{0}}$ is the ratio of the wet particle radius to the dry particle radius, representing the effect of relative humidity on aerosol refractive index.

The empirical relationship between $\frac{r(r h)}{r_{0}}$ and relative humidity can be expressed as [26]:

$$
\frac{r(r h)}{r_{0}}=(1-r h)^{-\frac{1}{d}}-(1-60 \%)^{-\frac{1}{d}}+1,
$$

where $d=3.5$.

The extinction efficiency factor caused by sea salt aerosol can be obtained as follows:

$$
Q_{e x t}=\frac{\lambda^{2}}{2 \pi} \sum_{n=1}^{\infty}(2 n+1) \operatorname{Re}\left(a_{n}+b_{n}\right) .
$$

Figure 4 indicates the relationship between the radius of sea salt particles and extinction efficiency factor. As depicted in the figure, the extinction efficiency factor increases with the increment of particle radius, where the ocean atmospheric transmission distance is $10 \mathrm{~km}$ and the relative humidity is $80 \%$. The extinction coefficient of sea salt particles plays an important role in the analysis of the influence of sea salt particles on ocean atmospheric CVQKD. In accordance with Mie scattering theory, the extinction coefficient is [19]:

$$
\beta_{\text {ext }}=\pi \int_{r_{1}}^{r_{2}} r^{2} Q_{e x t} f(r) d r .
$$

$f(r)$ is particle radius distribution function, $r_{1}, r_{2}$ is the minimum and maximum radius of particles respectively. The purpose of this paper is to analyze the effects of size of sea salt particles and relative humidity on ocean atmospheric CVQKD. Therefore, only single particle radius distribution is considered, and then $\beta_{\text {ext }}$ is expressed as: 


$$
\beta_{\text {ext }}=\pi r^{2} Q_{e x t} N
$$

$N$ is the number of particles for per unit volume. In this paper we set it as $N=10^{15} \mathrm{~m}^{-3}$ [27].

For ocean atmospheric CVQKD, the extinction of the beam and the distance of transmission act on the transmittance together. The transmittance can be expressed as [8]:

$$
T=e^{-\beta L}
$$

The total extinction coefficient comprises the aerosol scattering, aerosol absorption, molecular scattering, and molecular absorption terms. Among the many contributors to the extinction in atmosphere, we will focus here only on the sea salt particles, so we set scattering and absorption of the other aerosol and molecular as fixed value. Therefore, the extinction coefficient can be shown as:

$$
\beta=\beta_{\text {ext }}+\beta_{0}
$$

$\beta_{0}$ is the extinction of other aerosols and molecules, $\beta_{0}=10.3 \times 10^{-5}[8]$.

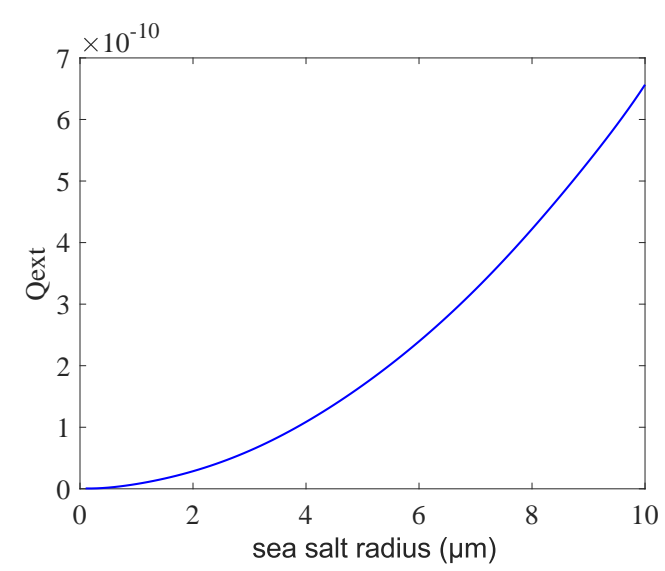

Figure 4. The relationship between particle radius and extinction coefficient. The transmission distance of sea surface is $10 \mathrm{~km}$ and the relative humidity is $80 \%$.

\section{Performance Analysis}

In this section, we analyze the performance of CVQKD protocol based on the previous research of atmospheric sea salt particles aerosol model. We apply Matlab simulations to estimate performance. According to the practical application, the assumption is that one party is on the shore and the other is on the reef when the key is transferred between the two parties. Here we only consider the attenuation caused by sea salt aerosol; conveniently, the attenuation caused by other particles is considered as fixed value. Moreover, excess noise $\varepsilon=0.03$ in SNU, $\lambda=550 \mathrm{~nm}$. In addition, we set $\beta_{1}=0.9, \eta=0.6$, simply.

First, we analyze the effects on secret key rate of sea salt radius and relative humidity under the scenario that the distance between shore-based and sea surface is invariable. The panel in Figure 5a plots the relationship between relative humidity and secret key rate, with the radius of sea salt particles being constant $r=0.5 \mu \mathrm{m}$. Figure $5 \mathrm{c}$ depicts the relationship between the radius of sea salt particles and the secret key rate when the relative humidity is $80 \%$. Secret key rate as a function of particle radius and relative humidity is illustrated in Figure 5e, where the distance of sea surface is $1 \mathrm{~km}, 2 \mathrm{~km}$, $3 \mathrm{~km}, 5 \mathrm{~km}, 8 \mathrm{~km}$, and $10 \mathrm{~km}$, respectively. As we can notice from the figures, when the distance between shore-based and sea surface remains unchanged, the larger the ocean transmission distances, the smaller the secret key rate. Furthermore, the secret key rate decreases with the increase of the radius of sea salt particles. Compared to the distance of ocean transmission with $1 \mathrm{~km}, 2 \mathrm{~km}, 3 \mathrm{~km}$, and $5 \mathrm{~km}$, when the distance of ocean transmission is $8 \mathrm{~km}$ and $10 \mathrm{~km}$, the secret key rate decreases to 0 earlier with the increase of particle radius. In addition, the secret key rate lessens with the increase of 
relative humidity because of the fact that the sea salt particles are hygroscopic particles. The change of relative humidity will cause the variation of particle radius, ultimately contributing to the decrease of the secret key rate. Nevertheless, the decrease in secret key rate is not as dramatic as that caused by particle radius.

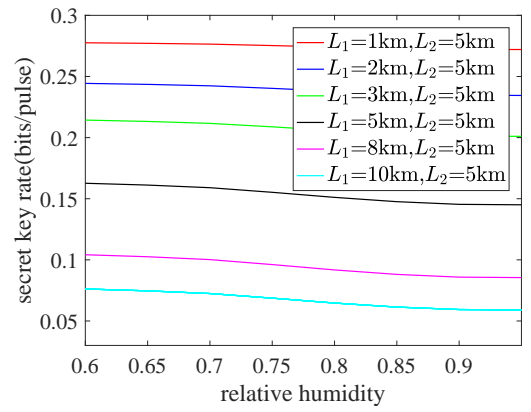

(a)

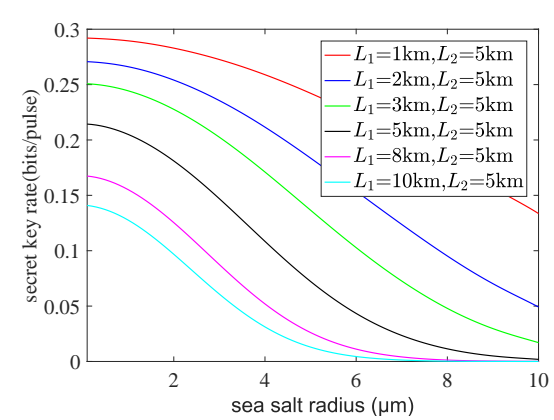

(c)

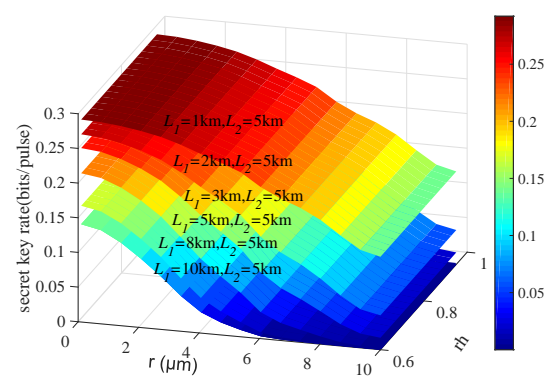

(e)

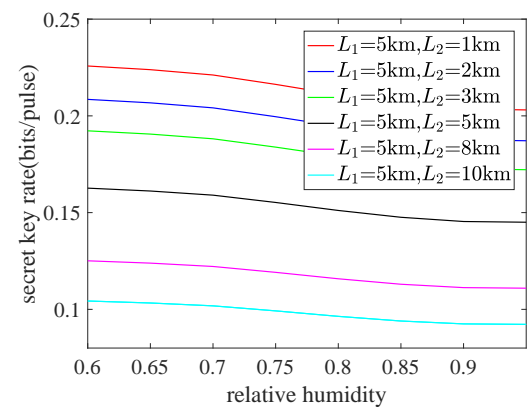

(b)

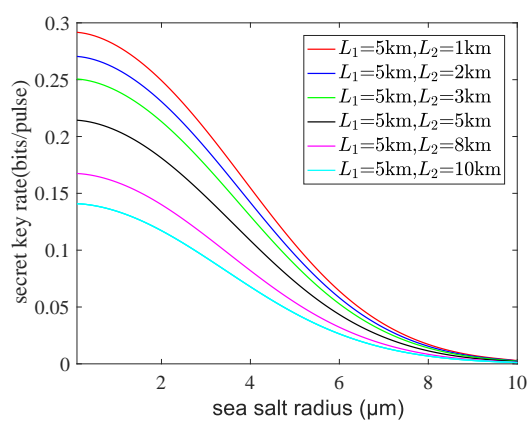

(d)

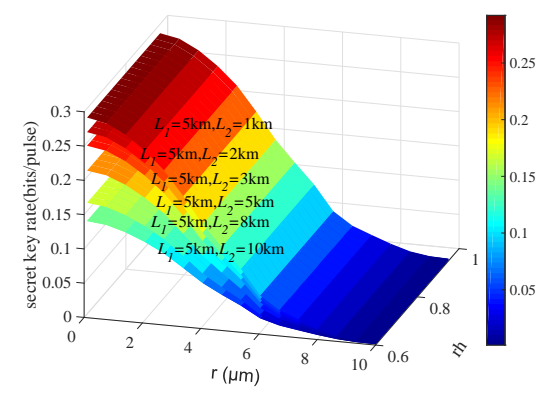

(f)

Figure 5. (a) The relationship between secret key rate and relative humidity, where the distance of shore-based is invariable and the sea salt radius is $0.5 \mu \mathrm{m}$. (b) Secret key rate versus relative humidity, where the distance of sea surface is invariable and sea salt radius is $0.5 \mu \mathrm{m}$. (c) Secret key rate versus sea salt radius, where the distance of shore-based is invariable and relative humidity is $80 \%$. (d) The relationship between secret key rate and sea salt radius, where relative humidity is $80 \%$. (e) Behavior of the secret key rate in terms of relative humidity and sea salt radius, when the distance of sea surface is $1 \mathrm{~km}, 2 \mathrm{~km}, 3 \mathrm{~km}, 5 \mathrm{~km}, 8 \mathrm{~km}$, and $10 \mathrm{~km}$, while the distance of shore-based is invariable. (f) Secret key rate as a function of relative humidity and sea salt radius, where the distance of shore-based is $1 \mathrm{~km}, 2 \mathrm{~km}, 3 \mathrm{~km}, 5 \mathrm{~km}, 8 \mathrm{~km}$, and $10 \mathrm{~km}$, respectively.

Second, we analyze the effects on secret key rate of sea salt radius and relative humidity under the scenario that the distance between reef and sea surface is invariable. In Figure $5 b$, the secret key rate as a function of relative humidity is plotted, when the distance of sea surface is fixed and the sea salt radius is $0.5 \mu \mathrm{m}$. The panel in Figure $5 \mathrm{~d}$ shows the relationship between secret key rate and sea 
salt radius; here relative humidity is $80 \%$. Figure $5 \mathrm{f}$ depicts the behavior of the secret key rate in terms of relative humidity and sea salt radius. As illustrated in the figures, we can find that with distance of ocean transmission unchanged, both the increase of total distance of transmission and the distance of shore-based will lead to the decrease of secret key rate. The reason for it is that the extinction coefficient is highly involved with the distance of transmission. The extinction effect is increasing with the soar of distance, which leads to the reduction of secret key rate. Similar to the case of fixed shore-based distance, the secret key rate decreases with the increase of relative humidity; however, the decline is not dramatic.

The numerical simulation demonstrates that the sea salt particles radius and relative humidity are closely related to the performance of ocean atmospheric CVQKD. Therefore, the role of sea salt could not be neglected. A study on the influence of sea salt particles on CVQKD protocol also provides a vigorous basis for evaluating CVQKD protocol in complex atmospheric environment.

\section{Conclusions}

In this paper, based on Mie scattering theory and atmospheric CVQKD model, the effects of sea salt particles on atmospheric channel CVQKD protocol are analyzed. Considering the practical application, the transmission channel is divided into two parts: the shore-based atmospheric channel and the ocean atmospheric channel. We implement the numerical simulation under the scenario of fixed different channels respectively. Consequences demonstrate that the radius and relative humidity of sea salt particles correspond to the performance of CVQKD protocol. No matter which part of channel distance we choose to fix, the secret key rate will decrease when the unfixed channel distance is increased. Simultaneously, with the increase of particle radius and relative humidity, the secret key rate also shows a downward trend. The results of this paper could lay a solid foundation for the establishment of shore-to-reef atmospheric CVQKD model and thus provide a basis for evaluating the performance of ocean atmospheric CVQKD protocol.

Author Contributions: Conceptualization, methodology, analysis, writing—review, and editing: S.Z., C.X., C.Z., X.W., J.Y., H.Z., W.B.

Funding: This research was funded by the National Nature Science Foundation of China grant number 61505261.

Conflicts of Interest: The author declares no conflicts of interest.

\section{Abbreviations}

The following abbreviations are used in this manuscript:

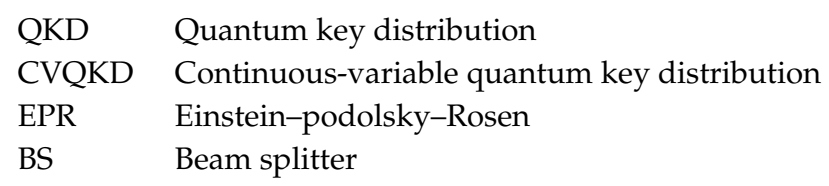

\section{References}

1. Bennett, C.H. Teleporting an unknown quantum state via dual classical and Einstein-Podolsky-Rosen channels. Phys. Rev. Lett. 1993, 70, 1895. [CrossRef] [PubMed]

2. Gisin, N.; Ribordy, G.; Tittel, W.; Zbinden, H. Quantum cryptography. Rev. Mod. Phys. 2001, 74, 145-195. [CrossRef]

3. Xuan, Q.D.; Zhang, Z.; Voss, P.L. A $24 \mathrm{~km}$ fiber-based discretely signaled continuous variable quantum key distribution system. Opt. Express. 2009, 17, 24244-24249. [CrossRef] [PubMed]

4. Qu, Z.; Djordjevic, I.B.; Neifeld, M.A. RF-subcarrier-assisted four-state Continuous variable QKD based on coherent detection. Opt. Lett. 2016, 41, 5507-5510. [CrossRef] [PubMed]

5. Schmitt Manderbach, T. Experimental demonstration of free-space decoy-state quantum key distribution over 144 km. Phys. Rev. Lett. 2007, 98, 010504. [CrossRef] [PubMed]

6. Semenov, A.A.; Vogel, W. Quantum light in the turbulent atmosphere. Phys. Rev. A 2009, 80, 92. [CrossRef] 
7. Usenko, V.C.; Heim, B.; Peuntinger, C. Entanglement of Gaussian states and the applicability to quantum key distribution over fading channels. New J. Phys. 2012, 14, 93048. [CrossRef]

8. Wang, S.Y.; Huang, P.; Zeng, G.H. Atmospheric Efects on Continuous-Variable Quantum Key Distribution. New J. Phys. 2018, 20, 083037. [CrossRef]

9. Yu, D.; Semenov, A. A.; Vogel, W. Toward global quantum communication: Beam wandering preserves nonclassicality. Phys. Rev. Lett. 2012, 108, 220501.

10. Heim, B.; Peuntinger, C.; Killoran, N. Atmospheric continuous-variable quantum communication. New J. Phys. 2014, 16, 113018. [CrossRef]

11. Elser, D.; Bartley, T.; Heim, B.; Wittmann, C.; Sych, D.; Leuchs, G. Feasibility of free space quantum key distribution with coherentpolarization states. New J. Phys. 2009, 11, 045014. [CrossRef]

12. Gókce, M.C.; Baykal, Y.; Uysal, M. Aperture averaging in multiple-input single-output free-space optical systems using partially coherent radial array beams. J. Opt. Soc. Am. A 2016 , 33, 1041-1048. [CrossRef] [PubMed]

13. Ricklin, J.C.; Hammel, S.M.; Eaton, F.D.; Lachinova, S.L. Atmospheric channel effects on free-space laser communication. J. Opt. Fiber Commun. Rep. 2006, 3, 111. [CrossRef]

14. Song, Z.F. The transmission of light in turbulent atmosphere. In Applied Atmospheric Optics; Song, Z.F., Tao, G.Q., Eds.; China Meteorological Press: Beijing, China, 1990; pp. 67-100.

15. Shi, P.; Zhao, S.C.; Gu, Y.J.; Li, W.D. Channel analysis for single photon underwater free space quantum key distribution. J. Opt. Soc. Am. A 2015, 32, 349-356. [CrossRef]

16. Xu, H.B.; Zhou, Y.Y.; Zhou, X.J.; Wang, L. Performance analysis of air-water quantum key distribution with an irregular sea surface. Optoelectron. Lett. 2018, 14, 216-219. [CrossRef]

17. Xie, C.L.; Guo, Y.; Wang, Y.J.; Huang, D.; Zhang, L. Security Simulation of Continuous Variable Quantum Key Distribution over Air-to-Water Channel Using Monte Carlo Method. Chin. Phys. Lett. 2018, 35, 090302. [CrossRef]

18. Guo, Y.; Xie, C.L.; Zeng, G.H. Channel-parameter estimation for satellite-to-submarine continuous-variable quantum key distribution. Phys. Rev. A 2018, 97, 052326. [CrossRef]

19. Wang, Y.J.; Fan, C.Y.; Wei, H.L. Attenuation of laser beam in seawater. In Laser Beam Propagation and Applications through the Atmosphere and Sea Water; Zhou, S.H., He, W.Z., Eds.; National Defense Industry Press: Beijing, China, 2015; pp.99-108.

20. Hansen, J.E.; Travis, L.D. Light scattering in planetary atmospheres. Space Sci. Rev. 1974, 16, 527-610. [CrossRef]

21. Guo, H.; Li, Z.Y.; Peng, X. Contiounous variable protocol. In Quantum Cryptography; Zhou, S.H., He, W.Z., Eds.; National Defense Industry Press: Beijing, China, 2016; p. 318364.

22. Diamanti, E.; Leverrier, A. Distributing Secret Keys with Quantum Continuous Variables: Principle, Security and Implementations. Entropy 2015, 17, 6072-6092. [CrossRef]

23. Mie, G. Beiträge zur Optik trüber Medien, speziell kolloidaler Metallösungen. Ann. Phys. 1908, 330, $377-445$. [CrossRef]

24. James, A.L.; Gouesbet, G. Generalized Lorenz-Mie theory and applications. J. Quant. Spectrosc. Radiat. Transf. 2009, 110, 800-807.

25. Horvath, H. Gustav Mie and the scattering and absorption of light by particles: Historic developments and basics. J. Quant. Spectrosc. Radiat. Transf. 2009, 110, 787-799. [CrossRef]

26. Sun, J.Q. The relationship between visibility and relative humidity. Acta. Meteor. Sin. 1985, 43, $230-234$.

27. Liang, T.T.; Marcelo, C.; Yu, X.P. Sea salt aerosol deposition in the coastal zone: A large eddy simulation study. Atmos. Res. 2016, 180, 119-127. [CrossRef]

(C) 2019 by the authors. Licensee MDPI, Basel, Switzerland. This article is an open access article distributed under the terms and conditions of the Creative Commons Attribution (CC BY) license (http://creativecommons.org/licenses/by/4.0/). 